Annuaire du Collège de France 2017-2018

\title{
Histoire contemporaine du monde arabe
}

\section{Henry Laurens}

\section{OpenEdition}

Journals

Édition électronique

URL : https://journals.openedition.org/annuaire-cdf/15619

DOI : 10.4000/annuaire-cdf.15619

ISBN : 978-2-7226-0572-5

ISSN : 2109-9227

Éditeur

Collège de France

Édition imprimée

Date de publication : 30 décembre 2020

Pagination : 403-413

ISBN : 978-2-7226-0516-9

ISSN : 0069-5580

Référence électronique

Henry Laurens, "Histoire contemporaine du monde arabe », L'annuaire du Collège de France [En ligne],

118 | 2020, mis en ligne le 01 avril 2021, consulté le 22 août 2022. URL : http://

journals.openedition.org/annuaire-cdf/15619; DOI : https://doi.org/10.4000/annuaire-cdf.15619 


\title{
HISTOIRE CONTEMPORAINE DU MONDE ARABE
}

\author{
Henry LAURENS \\ Professeur au Collège de France
}

Mots-clés : Orient, Occident, Première Guerre mondiale, Moyen-Orient, colonialisme, Empire ottoman

La série de cours et séminaires «Les crises d'Orient : le Moyen-Orient à partir de 1914 » est disponible, en audio, sur le site internet du Collège de France (https:// www.college-de-france.fr/site/henry-laurens/course-2017-2018.htm).

\section{ENSEIGNEMENT}

\section{COURS - LES CRISES D'ORIENT : LE MOYEN-ORIENT À PARTIR DE 1914}

Le cours de l'année 2017-2018 commence à partir de l'année 1914 et aborde l'histoire politique du Moyen-Orient comme objet géopolitique. Cette question s'inscrit dans la continuité du travail du professeur Henry Laurens publié en 2017 et intitulé Les Crises d'Orient (1768-1914). Le but de cet enseignement est de montrer que la source des violences réside davantage dans la géopolitique que dans la culture. À cet égard, l'exemple le plus éloquent par ses échos contemporains est celui de l'Allemagne de Guillaume II qui a encouragé un vaste projet de jihad contre la France et l'Angleterre, connu sous le nom du «jihad made in Germany». En dépit des travaux des émissaires allemands qui sont allés jusqu'en Afghanistan, ce projet a échoué.

D’une certaine façon, la Grande Guerre pourrait être considérée comme une crise d'Orient ayant pris une dimension pluricontinentale. En ce qui concerne les causes de la guerre, certains historiens comme Pierre Renouvin ont fait la distinction entre les causes immédiates comme la gestion diplomatique de la crise engendrée par l'attentat de Sarajevo et les causes dites « profondes » telles que l'hostilité franco-allemande due à la perte par la France de l'Alsace-Lorraine, la rivalité navale anglo-allemande et l'affirmation des nationalismes dans tous les pays d'Europe. Pour la question d'Orient, la chronologie abandonne la délimitation 1914-1918, il s'agit d'une guerre de dix ans (1912-1922), allant des guerres balkaniques à la guerre gréco-turque, voire de onze ans si la guerre italo-ottomane de 1911 est prise en compte. 
La thèse principale avait déjà été développée il y a près d'un siècle par l'historien britannique Arnold Toynbee: la question d'Orient est représentée par la confrontation, dans cette région du monde, des puissances occidentales autour de différents intérêts économiques, stratégiques, coloniaux, etc. Par ailleurs, la question de l'occidentalisation des sociétés et ainsi l'adoption du modèle universel de l'Étatnation s'imposent. Ceux qui avant 1914 étaient considérés comme des communautés au sens générique du terme deviennent à partir de 1919 des minorités. Autrement dit, le vocabulaire des traités concernant l'Europe centrale et orientale de 1919-1920 a été appliqué sur le Proche-Orient.

Cet enseignement qui s'intéresse au facteur oriental dans la crise de l'été 1914 permet une compréhension détaillée du jeu des puissances, qu'elles soient locales, régionales ou internationales. Dans leurs oppositions d'intérêts, nous pouvons constater la constance des ingérences et des implications. De ce point de vue-là, il n'existe pas un acteur qui ne se pense pas en termes de configuration géopolitique du système d'alliance. Ce cours se focalise donc sur la situation ottomane pendant cette guerre ; le gouvernement jeune-turc avait une bonne perception des intentions russes et ne pouvait pas avoir confiance dans les assurances qui lui seraient prodiguées par les belligérants concernant le respect de l'intégrité du territoire de l'Empire en cas d'adoption d'une position de neutralité par celui-ci. Pour les Jeunes-Turcs, la France et la Grande-Bretagne étaient les pays les plus engagés dans la domination de l'économie ottomane et la Russie était l'ennemi héréditaire aux dangereux desseins. Si les ambitions de l'Allemagne allaient plutôt dans le sens d'un protectorat, les Jeunes-Turcs n'étaient pas dupes de ces ambitions, mais considéraient qu'il était d'une nécessité vitale de disposer d'un allié puissant pour éviter un isolement qu'ils ont bien connu dans les guerres précédentes. Officiellement, l'Empire ottoman a adopté une position de neutralité le 3 août 1914, mais, dès le 2 août, un traité secret avait été conclu avec l'Allemagne. Si après la fermeture des détroits (à cause de la guerre italo-ottomane) et l'abolition des capitulations, la politique de neutralité armée paraît la plus favorable pour les Ottomans, Berlin exige une entrée en guerre. Le 27 octobre 1914, la flotte ottomane entre en mer Noire et entame les hostilités le 29 octobre contre les Russes en les accusant d'avoir ouvert le feu les premiers. Cette action s'est produite contre l'avis de la majorité du gouvernement ottoman. Après la défaite, les responsables ottomans feront porter sur Enver Pacha seul la responsabilité de l'entrée en guerre en collusion avec les Allemands. Si la Russie a poussé à la guerre en juillet 1914 - car elle disposait pour la première fois depuis 1814 d'alliés de première importance - l'Empire ottoman avait désespérément besoin d'un allié puissant pour assurer sa survie face à la Russie et pour lui c'était bien une lutte pour la survie qui s'est engagée en novembre 1914.

Pour les décideurs allemands, le jihad ${ }^{1}$ encouragé durant la crise de l'été 1914 est l'un des principaux atouts de l'Empire ottoman, d'où l'importance de conclure une

1. Le «jihad made in Germany », expression popularisée par l'orientaliste hollandais Snouck Hurgronge dans un article publié au début de 1915 et traduit rapidement en anglais, a été inspirée par le baron Max von Oppenheim (1860-1946), un membre de la famille des banquiers juifs Oppenheim qui se sont convertis au christianisme. Profitant de sa fortune personnelle, il a parcouru le Proche-Orient et est devenu un expert réputé des tribus bédouines ainsi qu'un archéologue. De 1896 à 1909, il est attaché à la légation d'Allemagne au Caire et, de ce poste 
alliance. L'Empire ottoman, de son côté, avait plaidé, face aux menaces européennes, pour une solidarité islamique en particulier durant les dernières décennies du $\mathrm{XIX}^{\mathrm{e}}$ siècle. Les progrès des communications et la diffusion de l'imprimé ont par ailleurs établi un monde musulman comme espace d'opinion. Cependant, les actions réelles de panislamisme n'ont eu que des résultats relativement médiocres; Abdülhamid II, $34^{\mathrm{e}}$ sultan ottoman, s'en est servi pour financer le chemin de fer du Hedjaz par souscription publique levée en partie à l'extérieur de l'Empire ottoman. Les Jeunes-Turcs ont de même levé des fonds auprès des musulmans de l'Empire russe pour l'achat des deux fameux Dreadnoughts en Grande-Bretagne.

La Grande Guerre est bien évidemment une guerre européenne et ses premiers enjeux concernent le continent européen. Cependant, les principales puissances européennes ont des empires coloniaux. Ces derniers en plus du lancement du «jihad made in Germany » ont pour double conséquence de faire de la domination du monde musulman indépendant l'un des grands enjeux de la guerre et en même temps de promouvoir les mouvements nationaux à l'intérieur de cet espace, une double conséquence qui s'avère finalement contradictoire. Autrement dit, cette guerre a aussi pour enjeu l'islam et son avenir dans une interaction intense et permanente avec les terribles convulsions du monde occidental, appelé « le monde civilisé ».

Le professeur Henry Laurens consacre dans son cours une partie aux échanges de correspondances entre le chérif Hussein et le haut-commissaire britannique au Caire sir Henry McMahon qui auraient abouti à la constitution d'un royaume arabe de Hussein composé de la péninsule arabique à l'exclusion de la Mésopotamie (réservée aux Britanniques) et de la côte syrienne allant d'Alep à la mer Morte (réservée à la France). En contrepartie, un soulèvement arabe contre l'Empire ottoman pour libérer les provinces arabes sous domination turque partira du Hedjaz. Par ailleurs, la négociation entre le représentant français Georges-Picot et le Britannique sir Marc Sykes est chargée de définir la frontière entre la Syrie et l'Arabie. L'accord, qui a été transmis par Sykes et Picot aux autorités, est donc un accord d'échange de lettres entre sir Edward Grey (ministre britannique des Affaires étrangères) et Paul Cambon (ambassadeur de France à Londres), d'où l'appellation dans les archives à l'époque de l'accord Cambon-Grey et non pas de l'accord Sykes-Picot. À partir de 1917, les Britanniques commencent la conquête de la Palestine et approuvent la fameuse déclaration Balfour à la séance du 2 novembre 1917 du cabinet de guerre. Cette déclaration envisage ainsi l'établissement en Palestine d'un Foyer national pour le peuple juif. Ce texte qui reconnaissait l'existence d'un peuple juif et refusait cette qualité de «peuple» aux «communautés non juives » a dénié à ces dernières tout droit politique.

La révolte arabe partie du Hedjaz en juin 1916 avait au départ une orientation nettement islamique contre le modernisme des Jeunes-Turcs. L'action des conseillers britanniques comme T.E. Lawrence sur le terrain et Sykes à Londres l'avait infléchie dans un sens plus moderne de nationalisme arabe pour l'inscrire dans le droit des peuples à disposer d'eux-mêmes. Cette révolte n'avait pas les moyens d'ébranler le front ottoman, mais elle a joué un rôle militaire conséquent. Son importance réside d'abord dans son rôle politique dans le cadre du droit des peuples à disposer d'euxmêmes. Il est difficile de négliger sa place dans l'histoire puisque son personnel

d'observation, il a multiplié les rapports sur la situation de l'islam de l'Atlantique à la Chine et s'est fait de multiples amis chez les notables musulmans du Proche-Orient. Il y préconise un soutien de l'Allemagne à un jihad contre les puissances coloniales. 
militaire et civil en 1918 comprend une bonne partie de la classe politique arabe de l'entre-deux-guerres. L' «homme malade » a quand même pu mobiliser dans des conditions des plus épouvantables environ deux millions d'hommes, ce qui montre qu'il n'était pas si déliquescent que cela. La disparition de l'Empire ottoman s'accompagne d'un démantèlement de l'appareil d'Etat, d'un ensemble organisé et centralisé capable de mobiliser des ressources et de lancer une grande entreprise collective. Cet effondrement a évidemment créé des successions d'États homogènes de population, ce qui a engendré, aussi bien dans le Caucase que dans les Balkans, au Proche-Orient, etc., ce qui a été appelé des «terres de sang », c'est-à-dire des espaces dans lesquels les constructions étatiques étaient faites sur la base d'épurations ethniques, de déplacements forcés de populations, voire de génocides. L'Anatolie a été une terrible terre de sang durant la Première Guerre mondiale et les années qui ont suivi. Par conséquent, il est possible d'observer une relative homogénéisation ethnique puisqu'il reste maintenant encore, face à face, les Kurdes et les Turcs en Anatolie avec des violences qui se poursuivent jusqu' aujourd'hui.

Une lecture commode des négociations diplomatiques de l'après-guerre évoque une forme de diktat franco-britannique qui aurait imposé à des populations impuissantes une division géographique du Proche-Orient, que l'on désigne généralement sous l'étiquette réductrice «Sykes-Picot ». Les principaux acteurs diplomatiques, y compris de haut rang, ignorent la rencontre privée en décembre 1918, entre Georges Clemenceau et Lloyd Georges, pendant laquelle Jérusalem et Mossoul sont données aux Britanniques dans la perspective d'un accord pétrolier qui permettrait à la France d'avoir sa part du pétrole de la Mésopotamie. Ces mêmes acteurs se lancent donc dans des stratégies destinées à obtenir le maximum de gains. Du côté britannique, T.E. Lawrence a joué un rôle important et s'est fait le conseiller à la fois des décideurs de son pays et de l'émir Faysal. Il organise une rencontre entre Weizmann et Faysal dont les restitutions historiques de l'événement sont si différentes qu'il est légitime de penser que Lawrence a manipulé les traductions.

À l'automne 1919, à la conférence de Deauville, les Britanniques décident d'évacuer la Syrie et de répartir leurs troupes en Palestine en raison des difficultés financières et de l'épuisement de leurs troupes. Les Français qui disposent d'une puissante armée coloniale grâce à l'application de la conscription - ce qui les différencie des Britanniques - bénéficient d'une situation favorable qui leur permet de relever les troupes britanniques. À partir de ce moment, le système des mandats doit s'installer mais il reste à parfaire la question politique de la division desdits mandats et leur répartition territoriale, ce qui est achevé à la conférence de San Remo qui se tient du 19 au 26 avril 1920. Celle-ci réunit les six puissances du Commandement suprême allié (France, Grande-Bretagne, Italie, Belgique, Japon, Grèce) tandis que les États-Unis sont présents sous forme d'observateurs. Faysal a refusé d'y assister et une délégation arabe n'est pas admise aux débats. La discussion sur le Proche-Orient a lieu les 24 et 25 avril où il est admis que les mandats A seront attribués à la France et à la Grande-Bretagne. L'essentiel des débats porte sur un essai d'interprétation de la déclaration Balfour et l'élaboration du traité de paix avec l'Empire ottoman. Les procès-verbaux ${ }^{2}$ des délégations participantes à la conférence

2. Contrairement à ce qui est écrit abondamment y compris par des auteurs sérieux, il n'y a pas de «traité » ou «d'accord» de San Remo, mais seulement des procès-verbaux tenus par les délégations et la rédaction des clauses du traité. 
diffèrent largement comme l'a déjà explicité le professeur Henry Laurens dans son ouvrage Orientales $\mathrm{III}^{3}$. Finalement, la carte définitive est donc celle de San Remo ; pour les frontières de la Palestine, techniquement du moins, on s'est servi d'un atlas biblique. Les Français ne souhaitaient pas avoir de colonie juive dans leur territoire d'autant plus que l'hostilité de la Troisième République au sionisme est complète : c'est une invention allemande utilisant des Russes communistes au service de la Grande-Bretagne et contraire à l' assimilationnisme de l'Alliance israélite universelle.

Au Proche-Orient, les nouvelles de France, sur ce qui se discute à la conférence de paix, ne font que renforcer le mécontentement. En Palestine, les notables et les jeunes éduqués rejettent toute concession, y compris celle de la simple égalité des droits avec les sionistes et exigent une Palestine arabe indépendante. En Syrie, les jeunes éduqués, réunis dans le mouvement clandestin al-Fatat (les jeunes Arabes) radicalisent aussi leurs positions contre les projets français.

La conférence de San Remo avait terminé la rédaction du traité avec les Ottomans. Signe des temps, le texte évoque à la fois la « Turquie » et le gouvernement ottoman. Ces derniers sont informés de son contenu, particulièrement dur, le 11 mai 1920 : sur le plan territorial, la Grèce reçoit la plus grande partie de la Thrace, Constantinople, les rivages de la mer de Marmara et les détroits sont démilitarisés. La navigation est libre sous le contrôle d'une commission internationale et les Puissances peuvent y intervenir pour protéger les minorités ethniques. Au Sud, la Cilicie est dans le mandat français et le vilayet de Mossoul dans le mandat britannique. Le Dodécanèse passe définitivement à l'Italie.

À la mi-mars 1921, il est clair que le traité de Sèvres est mort-né. Si le sultan se résigne à signer le traité, après deux mois de discussion, les nationalistes, dirigés par Mustafa Kemal considèrent qu'il était inadmissible de le signer et mènent à partir de l'Anatolie, une lutte acharnée contre le gouvernement de Constantinople et contre les puissances occupantes depuis juin 1919. La situation sur le terrain a mis fin aux projets arménien et kurde, il ne reste plus qu'un inévitable affrontement gréco-turc et les Puissances sont dans l'incapacité d'imposer une solution. La Grande-Bretagne, la France et l'Italie annoncent leur neutralité dans la guerre qui va reprendre. Les tergiversations diplomatiques ne cachent pas que le rapport de forces favorise de plus en plus les Turcs qui reçoivent de l'armement de la part de la Russie soviétique et accessoirement des Français et des Italiens qui ont obligeamment laissé des stocks lors de leurs évacuations.

Du côté de la Syrie, la bataille de Khan Maysaloun opposant Faysal et l'armée française en juillet 1920 avait montré l'impossibilité d'arriver à un accord entre un nationalisme arabe émergent et la politique impériale française. Tout en restant dans le discours de la politique musulmane, les autorités françaises considèrent maintenant le nationalisme arabe comme un danger, non seulement pour la France du Levant, mais aussi pour l'Afrique du Nord.

En outre, la création du Grand Liban était la récompense du ralliement des chrétiens à la politique française et la volonté de continuer l'expérience levantine dans un cadre plus national. En revanche, la Syrie est divisée en plusieurs États et territoires, aussi bien dans une politique de «diviser pour régner» que pour émanciper les non-sunnites de la domination sunnite. Finalement, le traité de Sèvres

3. Henry LaURens, Orientales III. Parcours et situations, Paris, CNRS Éditions, 2004, p. $177-206$. 
et la bataille de Maysaloun restent profondément inscrits dans la mémoire politique du Proche-Orient comme les deux revers principaux des nationalismes turc et arabe dont il faut éviter la réédition.

La seconde partie de l'année 1922 voit la concrétisation des nouvelles entités territoriales issues de la Première Guerre mondiale. La France et la Grande-Bretagne ne se sont pas opposées frontalement au kémalisme dans la mesure où ce dernier avait accepté dès le départ de ne pas revendiquer les anciennes provinces arabes de l'Empire ottoman. La signature du traité de Lausanne le 24 juillet 1923 constitue à la fois l'acte de décès de l'Empire et un immense succès qui donne à la Turquie un statut de totale égalité avec les puissances occidentales. Un autre exemple abordé pendant ce cours est celui du nationalisme égyptien ; la révolution de 1919 a montré qu'il n'était plus possible de s'en tenir à une politique des notables, puisque le Wafd, autour de Saad Zaghloul, avait la capacité d'agir sur la totalité du territoire égyptien.

À partir de 1923-1924, la stabilité tant espérée semble s'installer de façon durable, même si quelques remaniements territoriaux sont encore à venir. C'est le début du « moment britannique », celui d'une hégémonie qui constitue une sorte de parenthèse entre le pluricentrisme de la question d'Orient et le retour des compétitions internationales lors de la Seconde Guerre mondiale et surtout de la guerre froide. Les Français, quant à eux, n'ont aucune ambition d'élargir leur domaine et se sentent en position défensive face aux ambitions réelles ou supposées des Britanniques.

Ainsi, la région ne semble plus obéir à sa malédiction géopolitique d'être l'objet permanent des implications et des ingérences des divers acteurs. Son enjeu se transforme avec la disparition progressive de la fantomatique route de l'Inde et son remplacement progressif par les routes plus réelles du pétrole puisqu'il s'agit maintenant du «pays de l'or noir» tandis que l'enjeu palestino-sioniste prend une importance mondiale.

La violence au $\mathrm{XX}^{\mathrm{e}}$ siècle vient de l'Europe qui se bat sur plusieurs continents. Les conflits mondiaux sont donc des conflits européens. Le problème spécifique du monde arabe réside dans le fait qu'il est à la fois trop proche de l'Europe et trop près de Dieu, ce qui constitue une fusion détonante qui explique la violence d'aujourd'hui. En concevant son cours, le professeur Henry Laurens a été inspiré par une réflexion sur les événements les plus actuels : c'est à partir des déclenchements de la guerre civile syrienne et avant elle irakienne que la question qui se pose est celle de savoir s'il ne s'agissait pas d'un nouvel épisode d'un système politique qui s'était mis en place il y a plus de deux siècles.

\section{SÉMINAIRE - CULTURE POLITIQUE ARABE}

Le séminaire consacré à la culture politique arabe propose cette année encore un panorama des dynamiques sur un temps long. Ces éclairages inédits sur des développements récents, des aires géographiques ou encore des séquences particulières dans cette région ont été présentés par les meilleurs spécialistes de la question.

Plusieurs types de présentation ont été proposés; deux séminaires ont été consacrés à la Syrie, son histoire du $\mathrm{XIX}^{\mathrm{e}}$ au $\mathrm{XXI}^{\mathrm{e}}$ siècle, les grandes scansions qui modifient la trajectoire du pays ainsi qu'un développement sur un passé syrien mêlé à celui de l'Europe. 
Les intervenants ont été Jean-Pierre Filiu, Matthieu Rey, Hamit Bozarslan, Bernard Rougier, Nabil Mouline, Eberhard Kienle.

Une séance a mis l'accent sur la culture politique kurde au XXe siècle et notamment les trajectoires politiques après la chute de l'Empire ottoman. La société saoudienne, les enjeux de la succession ainsi que les jeux de pouvoir de la famille royale ont été également abordés et présentés en détail.

Deux séances ont traité de la question de l'islam politique et des questions du jihad sur deux aspects différents : une première intervention a traité des conditions de la naissance de l'islamisme sunnite à travers l'itinéraire d'Abdallah Azzam, connu pour son rôle dans le mouvement du jihad mondial. Une deuxième intervention a abordé l'avenir de la Syrie et de l'Irak entre résilience et résiliation après la défaite de l'État islamique.

Ces interventions variées ont à chaque fois donné lieu à des échanges avec les intervenants, portant sur les recherches exposées ou sur l'actualité la plus immédiate. Le séminaire - et le fait qu'il se tienne depuis 2016 en amphithéâtre Marguerite de Navarre en témoigne - a ainsi confirmé son ancrage comme lieu de rencontre et d'excellence sur le monde arabe.

\section{CONFÉRENCIER INVITÉ}

\section{Mohamed-Mahmoud Ould Mohamedou : Histoires croisées d'Al-Qaïda et de l'État islamique}

Conférence donnée par Mohamed-Mahmoud Ould Mohamedou, professeur à The Graduate Institute, Maison de la Paix, Genève (Suisse), le 29 novembre 2017

L'histoire des nouveaux groupes armés non étatiques que sont Al-Qaïda et l'État islamique (EI) est celle de l'auto-capacitation d'une violence politique contemporaine qui desserre la dimension spatiale des relations internationales et consacre la transnationalisation grandissante de ces entités. Dans ce contexte, l'avènement de l'EI s'inscrit dans un parcours de violence militarisée plus vaste entamé plus tôt par Al-Qaïda. Les micro-récits relatifs à ces deux groupes centrés sur leurs leaders et leur religiosité théâtralisée ont occulté les enseignements plus larges qu'il faut tirer concernant l'histoire de la violence politique à la fin du $X X X^{\mathrm{e}}$ siècle et au début du $\mathrm{XXI}^{\mathrm{e}}$ siècle. L'EI, comme Al-Qaïda, souffre d'un déficit analytique et d'une absence de conceptualisation. Les sciences sociales, généralement, et les relations internationales, plus précisément, ont, pour l'heure, été incapables de cerner la nature des importantes transformations systémiques - la militarisation de l'islamisme, l'indigénisation du transnationalisme et l'entreprenariat de violence post-moderne - introduites par ces deux groupes. Confinées à des approches sécuritaires, informées de façon culturaliste et distillées sur un mode médiatique, les analyses de ces nouvelles formes de violences dénotent un manque conceptuel. Le terrorisme est aujourd'hui avant tout révélateur d'un «moment historique » de ces mutations de la contestation dont l'essor a lieu sur l'arrière-plan d'une modernisation rapide et mondialisée. 


\section{RECHERCHE}

Les relations entre Orient arabe et Occident constituent toujours l'axe principal des recherches de la chaire. Des thématiques comme le suivi de trajectoires nationales (Égypte, Syrie, Palestine), les relations internationales, bilatérales (Palestine et Égypte ; Syrie et France) ou multilatérales (France et pays de la Méditerranée) restent au cœur de la production scientifique de la chaire.

\section{ACTIVITÉS DU PROFESSEUR}

J'ai été invité comme professeur à l'Université libre de Bruxelles, pour cinq séances, à l'automne 2017. J'ai également donné plusieurs conférences à Paris et en province, ainsi que des enseignements à l'université Saint Joseph de Beyrouth. J'ai participé à deux séminaires de la fondation Moulay Hicham et ai fait une mission en Russie en juin 2018.

J'ai publié Les Crises d'Orient. Question d'Orient et Grand Jeu (1768-1914), Paris, Fayard, coll. « Fayard Histoire », 2017.

\section{ÉQUIPE}

\section{Manon-Nour Tannous, chercheure associée à la chaire}

L'année 2017 - dans le cadre de son poste d'ATER au Collège de France - a largement été consacrée à finaliser son manuscrit, dans la perspective de la publication d'une version remaniée et augmentée de sa thèse de doctorat. L'ouvrage est paru aux PUF en octobre 2017 sous le titre Chirac, Assad et les autres, les relations franco-syriennes de 1946 à nos jours. Cette parution a donné lieu à plusieurs interventions sur les relations entre la France et la Syrie, tant sur le temps long que pour saisir les reconfigurations actuelles. Au sein du Collège de France, un travail collectif lui a permis de contribuer à l'ouvrage Méditerranées politiques, codirigé par Henry Laurens et Matthieu Rey. Le Collège de France - et en particulier la chaire Histoire contemporaine du monde arabe - a, par ailleurs, été l'institution d'accueil des séminaires doctoraux organisés par le Cercle des chercheurs sur le Moyen-Orient, association qu'elle préside aujourd'hui. Mes recherches actuelles portent, d'une part, sur les acteurs non étatiques de la relation diplomatique entre l'Europe et l'Orient arabe, d'autre part, sur le conflit syrien depuis 2011. Parallèlement, M.-N. Tannous a poursuivi cette année ses enseignements universitaires sur le Moyen-Orient et plus généralement sur les enjeux stratégiques contemporains, à Sciences Po Paris et l'université Paris X-Nanterre.

Publications :

TANnous M.-N., Chirac, Assad et les autres, les relations franco-syriennes depuis 1946, Paris, PUF, 2017.

Bonneville A.-C., Chiffoleau S., Neveu N., Rey M., Tannous M.-N. et Turiano A.-L. (dir.), Le Moyen-Orient de 1876 à 1980, Paris, Atlande, 2017.

Tannous M.-N., « De Barcelone à Tunis : l'idée de Méditerranée en quête d'elle-même », in M. REY et H. LAURENS (dir.), Méditerranées politiques, Paris, PUF, 2017. 
Tannous M.-N., «De l'utilité d'un changement d'approche de la guerre en Syrie », Les Champs de Mars, vol. 30, n 1, 2018, p. 191-200.

TANNOUS M.-N., «Les ressources stratégiques de la rupture en diplomatie : comparaison des ruptures franco-syriennes », Études internationales, vol. 47, no 2-3, 2016, p. 219-239, DOI : 10.7202/1039544ar.

TANnous M.-N., «Le Conseil de sécurité et la colonisation israélienne », Revue générale de droit international public, vol. 121, $\mathrm{n}^{\mathrm{O}}$ 3, 2017.

TANNOUS M.-N., «"Tenir son rang”: la politique française à l'épreuve de la crise syrienne (2011-2015) », Critique internationale, vol. 74, nº 1, 2017, p. 117-136.

TanNous M.-N., «La France et l'approche multilatérale de la crise syrienne », Annuaire français des relations internationales, vol. XVIII, 2017, p. 333-347.

\section{Matthieu Rey, chercheur associé à la chaire}

L'année 2017 a été consacrée à deux projets d'ampleur. Le premier fut la remise à Cambridge University Press du manuscrit de thèse amendé, portant sur les mutations du système parlementaire en Syrie et en Irak de 1946 à 1963. Ce dernier a bénéficié d'un soutien de la part du Collège de France et est en cours d'évaluation. Le second porte sur la collecte de la documentation, son analyse et son exploitation pour produire une monographie sur la Syrie contemporaine depuis le XIX ${ }^{\mathrm{e}}$ siècle. En dehors de cela, ses fonctions de chargé de recherche au CNRS ont permis à M. Rey de renforcer les partenariats de son laboratoire à l'Iremam avec le Collège de France autour, entre autres, du programme 1979 : migrations des espérances. Ce dernier a débouché sur le dépôt d'un ERC consolidator grant, sous la direction de Malika Rahal. Cette collaboration entre le Collège de France et le CNRS s'est traduite aussi par la publication en 2017 d'un opus, Méditerranées politiques, conjointement dirigé avec Henry Laurens. Les autres thèmes d'interventions, de prises de parole et de publication portent sur le fait politique en Syrie et en Irak, les derniers bouleversements en cours en Syrie, sur la guerre froide.

Publications :

REY M., Histoire de la Syrie XIX ${ }^{e}-X X I^{e}$ siècle, Paris, Fayard, 2018.

Bonneville A.-C., Chiffoleau S., Neveu N., Rey M., Tannous M.-N. et Turiano A.-L. (dir.), Le Moyen-Orient de 1876 à 1980, Paris, Atlande, 2017.

Lemire V. (dir.), Crouzet G., Dumasy F. et Rey M., Le Moyen-Orient de 1876 à 1980, Paris, Armand Colin, 2016.

LaURens H. et Rey M. (dir.), Méditerranées politiques, Paris, PUF, coll. «La vie des idées », 2017.

REY M., «2003: A turning point in the formation of syrian youth », in M.M. AYYASH et R. HAJJ MousSA (dir.), Protests and Generations: Legacies and Emergences in the Middle East, North Africa and the Mediterranean, Leiden, Brill, 2017, p. 97-121.

REY M., «Dall'iraq al medio oriente : l'is e la creazione di una nuova entita politica », in M. Trentin (dir.), L'ultimo califfato. L'Organizzazione dello Stato islamico in Medio Oriente, Milan, il Mulino, 2017.

REY M., " "Domestiquer" la guerre froide au Moyen-Orient (1945-1961) : dynamiques externes et trajectoires irakienne et syrienne », Relations internationales, vol. 171, $\mathrm{n}^{\circ} 3,2017$, p. 69-84. 


\section{Jalila Sbaï, chercheure associée à la chaire}

J. Sbaï a consacré l'année à la préparation et la publication de son livre $L a$ Politique musulmane de la France. Un projet chrétien pour l'islam ? 1911-1954, paru aux éditions du CNRS en 2018, et elle travaille à un second volume intitulé Les Cours de Robert Montagne au Collège de France (1949-1954). Ses activités ont eu un volet diffusion et vulgarisation de la recherche en tant que membre du comité de rédaction du magazine Orient $X X I$.

Publications :

SBAÏ J., La Politique musulmane de la France Un projet chrétien pour l'islam ? 1911-1954, Paris, CNRS Éditions, 2018.

SBAI J., «Comment la France a traité l'islam et les musulmans. Des Lumières aux débats actuels », Orient XXI, 2017, https://orientxxi.info/magazine/comment-la-france-a-traite-1islam-et-les-musulmans, 1767.

\section{Valérie Stiegler, doctorante associée à la chaire}

L'année 2017 a été largement consacrée à la rédaction de sa thèse portant sur la politique méditerranéenne du président Georges Pompidou, sous la direction du professeur Henry Laurens et du professeur Alya Agan, à l'université Paris 1. Outre la rédaction de sa thèse, le travail de recherche de V. Stiegler s'est scindé en deux activités. Il s'agit d'abord de communications et de publications sur la politique française en Méditerranée. Elle a ainsi pu effectuer une communication lors du colloque organisé par l'institut Georges Pompidou sur «Jean Bernard Raimond, un diplomate en politique » qui s'est déroulé le 12 octobre 2017 à la bibliothèque polonaise (Paris). Par ailleurs, elle a organisé, dans le cadre d'une association de chercheurs sur le Moyen-Orient, le CCMO, des manifestations diverses : séminaires doctoraux, rencontre autour du droit et des réfugiés.

Publications :

STIEGLER V. et REY M., « Nords et Suds, vers une nouvelle régionalisation (1950-1970) », in M. ReY et H. LAURENS (dir.), Méditerranées politiques, Paris, PUF, 2017.

\section{François Ceccaldi, doctorant associé à la chaire}

Cette année a été consacrée essentiellement à la rédaction de sa thèse qui porte sur les mouvements d'opposition internes et à la question de la réforme de l'OLP à partir des années 1990. F. Ceccaldi a également dispensé des enseignements à Lyon 3 dans le cadre de la préparation des étudiants à l'épreuve d'histoire contemporaine de l'agrégation d'histoire et assuré des vacations à Sciences Po Paris en histoire du Moyen-Orient.

Publications :

CECCAldi F. et AlsajdeyA D., « Trump ou la diplomatie du choc. Les enjeux de Jérusalem capitale », La vie des idées, 19 décembre 2017, http://www.laviedesidees.fr/Trump-ou-ladiplomatie-du-choc.html.

CeCCAldi F., « La Palestine, des accords au conflit », La Vie des idées, 26 juin 2017, http:// www.laviedesidees.fr/La-Palestine-des-accords-au-conflit.html. 
CECCALDI F., «Le septième congrès du Fatah: entre affirmation d'autorité et perte de légitimité pour Mahmoud Abbas », Les Carnets de l'Ifpo, 22 mars 2017, https://ifpo. hypotheses.org/7463.

\section{Dima Alsajdeya, ATER à la chaire}

Entre septembre 2016 et août 2017, D. Alsajdeya occupait le poste de chargée de mission à l'Institut de recherches et d'études Méditerranée Moyen-Orient à Paris, où elle a pu organiser des manifestations diverses : colloques, conférences, séminaires, journées j'études etc. Depuis septembre 2017, elle est ATER à la chaire et son temps a été essentiellement consacré à sa thèse, qui porte sur le rôle et l'implication de l'Égypte de Moubarak dans les tentatives de règlement du conflit israélo-palestinien (1981-2011). Dans le cadre de ses recherches, elle a effectué, en avril 2017, un terrain de recherche en Palestine pour mener des entretiens avec des acteurs sur place. Parallèlement, elle a fait plusieurs interventions pour parler de la question de Palestine et des processus de paix.

Publications :

CecCAldi F. et Alsajdeya D., « Trump ou la diplomatie du choc. Les enjeux de Jérusalem capitale », La vie des idées, 19 décembre 2017, http://www.laviedesidees.fr/Trump-ou-ladiplomatie-du-choc.html.

ALSAJDEYA D., « Retour sur vingt-cinq ans de relations incertaines entre Israéliens, Égyptiens et Palestiniens », Confluences Méditerranée, vol. 100, n 1, 2017, p. 65-77.

ALSAJDEYA D., «Les conséquences socio-économiques de la construction du mur israélien en territoires palestiniens », Orients stratégiques, nº 4, 2016, p. 121-134. 
